Research Article

\title{
Evaluating the Role of Big Data in IIOT-Industrial Internet of Things for Executing Ranks Using the Analytic Network Process Approach
}

\author{
Xiaoqun Liao, ${ }^{1}$ Mohammad Faisal $\mathbb{D},{ }^{2}$ Qing QingChang $\mathbb{D},{ }^{3,4}$ and Amjad Ali ${ }^{5}$ \\ ${ }^{1}$ Information and Network Center, Xi'an University of Science and DSTechnology, Xi'an 710054, China \\ ${ }^{2}$ Department of Computer Science \& IT, University of Malakand, Chakdara, Pakistan \\ ${ }^{3}$ School of Information Management and Engineering, Shanghai University of Finance and Economics, Shanghai 200433, China \\ ${ }^{4}$ School of Information Management, Shanghai Linxin University of Accounting and Finance, 995 Shangchuan Road, \\ Pudong New District, Shanghai 201209, China \\ ${ }^{5}$ Department of Computer Science, University of Swat, Mingora, Pakistan
}

Correspondence should be addressed to Qing QingChang; changqingqing54@sina.com

Received 29 July 2020; Revised 2 September 2020; Accepted 14 September 2020; Published 20 October 2020

Academic Editor: Habib Ullah Khan

Copyright ( 92020 Xiaoqun Liao et al. This is an open access article distributed under the Creative Commons Attribution License, which permits unrestricted use, distribution, and reproduction in any medium, provided the original work is properly cited.

Due to the enhancements of Internet of Things (IoT) and sensors deployments, the production of big data in Industrial Internet of Things (IIoT) is increased. The accessing and processing of big data become a challenging issue due to the limited storage space, computational time, networking, and IoT devices end. IoT and big data are well thought-out to be the key concepts when describing new information architecture projects. The techniques, tools, and methods that help to provide better solutions for IoT and big data can have an important role to play in the architecture of business. Different approaches are being practiced in the literature for evaluating the role of big data in IIoT. These techniques are not handling the situations when complexity of dependency arises among parameters of the alternatives. The proposed research uses the approach of Analytic Network Process (ANP) for evaluating the role of big data in IIoT. The results show that the proposed research works well for evaluating the role of big data in IIoT.

\section{Introduction}

The developments in the field of data processing, Internet, and electronic communications have enabled the world to easy access to different physical devices. The whole world is covered with different devices embedded with the actuators and sensors. Huge bulk of data is produced from the communication of these heterogeneous devices which need to be researched for mining useful insights. These useful information and insights will play an important role in decision-making and optimum management or services and resources.

The integration of emerging trends, technologies, and applications of IoT in the industrial environment is leading toward the expansion and developments of IIoT. The IIoT is collection of smart devices and objects which sense, collect, process, and communicate real-time occurrences in the industrial setup. IIoT aims to achieve high efficiency of operation to manage industrial assets and increase the productivity of industry with the support of product customization. In the last few years, special attentions were given to the applications of IoT with emerging into the industry. Several IoT devices are brought to facilitate the human life. The applications of IoT technologies in industry of automation have been expended to the IIoT which supports Cyber Physic System in which human and machine interact [1]. Internet of Things has several applications in the daily life and has made life very easy. From industry to education, healthcare, and to other place, the IoT is mostly used. Internet of Medical Things is the advanced version of 
IoT which has a key role in healthcare. In healthcare, the devices are sometimes connected through heterogeneous environment with the support of different IoT devices.

IIoT takes help from the communication of IoT in the applications of business which mostly focus on the interoperability among machines. As the daily life objects and things connected to the Internet are increasing with the passage of time, which made the IoT be dynamic network of networks, more challenges such as dynamicity, heterogeneity, volume, and velocity of data makes the services of IoT inconsistent, incomplete, inaccurate, and incorrect results which resultant can make complexity for the applications of IIoT such as healthcare, wearable, smart transportation, and industry [2].

The contribution of the proposed research is to use the approach of Analytic Network Process for evaluating the role of big data in IIoT. The process of pairwise comparison was carried out for the criteria and available alternatives. The results show that the proposed research works well for evaluating the role of big data in IIoT.

The organization of the paper is as follows. Section 2 presents the related work to the role of big data in IIoT. Section 3 shows the research method of the proposed research. The results and discussions are shown in Section 4. The paper is concluded in Section 5.

\section{Related Work}

With the enhancement in IoT and sensors deployments, the production of big data in IIoT is increased. The accessing and processing of big data become a challenging issue due to the limited storage space, computational time, networking, and IoT device end. Researchers try to use different techniques and tools for evaluating and extracting meaningful insights from such data. Ur Rehman et al. [3] identified technologies of big data analytics, techniques, and algorithms used in the development of intellectual industrial Internet of Things systems. Classification and categorization taxonomy were devised for the literature based on significant parameters such as analytics tools, data sources, requirements, analytics techniques, and industrial applications. Different frameworks and case studies are presented to show the applications of big data analytics in various enterprises. Hadj Sassi et al. [4] proposed an architecture of big data and cognitive Internet of Things. The approach integrates the Data Lake and Data Warehouse and then defines the tool for collection of heterogeneous data. Sunhare et al. [5] presented a comprehensive and systematic review of the different data mining techniques employed in small- and large-scale IoT for formulating an intelligent environment. The system of cloud-based IoT big data mining is also discussed. Yao et al. [6] presented the applications of deep learning model for diagnosing gallbladder stone from Internet of Health Things (IoHT) big data. The research categorises the characteristics of gallstone for improving the presented model to determine the chemical compositions of gallstone. The model obtain smart healthcare data from IoT for supporting diagnose and recommendation of treatment of gallbladder stone with the aim to build smart IoHT.
Khan et al. [7] presented the concept of IIoT in a new way to help reader to understand the IIoT. The existing efforts made for research in the IIoT are described. The study highlights the enabling technologies for IIoT and the challenges to the IIoT. Gulati and Kaur [8] analysed the key opportunities integrated from the concept of SIoT into the industry with proposing reference architecture. An ontological model is designed to present the model from a semantic perspective. For the relationship management, an approach among manufacturing resources is introduced. The authors [1] presented the ideas of data management in IoT, the literature of data management in IoT, the most relevant solutions, and traced open research challenges. Aceto et al. [9] provide the detail description of the key technologies and approaches used in association to Healthcare 4.0, the key applications scenario, benefits, multidisciplinary challenges, and the derivations. Younan et al. [2] presented a study with comprehensive review of the existing challenges in the literature and recommending technologies for enabling the analysis of data and search in the future IoT search engines. Two case studies are presented to show promising growth on smartness and intelligence of applications of IoT based on the integration of information and communication technologies. The applications of smart phone enable the patients to know about their diseases after the analysis in the field of gynaecology and paediatrics [10]. Mobile computing services can be used in IoT by using services of mobile phones, apps, or through M-Health care system [11].

Feldner and Herber [12] presented a qualitative approach for evaluation of IPv6 for IIoT. Interviews were conducted with five experts of IPv6 users. The experimental results indicate the key challenges are (a) tool help and available libraries are immature, (b) user can configure the IP communication manually, and (c) complicated one-fitsall protocol is prevalent. Boyes et al. [13] presented the concept of IIoT and the association to the ideas such as cyber physical systems and Industry 4.0. IoT-related taxonomies were analysed and developed an analysis framework for IIoT that can be used to list and characterise the devices of IIoT when analysing security vulnerability and threats. Alexopoulos et al. [14] presented the IIoT architecture and its development details to support the industrial product service system life cycle. Ge et al. [15] presented a survey on the technologies of big data in different domains of the IoT for facilitating and stimulating sharing of knowledge across the domains of IoT. The similarities and differences among the technologies of big data in different domains with the reusability of technologies in the domain of IoT are discussed. Lin et al. [16] presented an approach of integrating sensing data from diverse sources and equipments to apply on IIoT. The industrial Micro Control Unit is connected to interface with actuator, data sources, and equipments. The experimental results show that IIoT can reduce the problem of heterogeneous protocol and database manufacturing data transmission.

Humayun et al. [17] presented a comprehensive report of the evolution, prevention, and mitigation of Ransomware in the context of IoT. Jiang [18] has presented an approach 


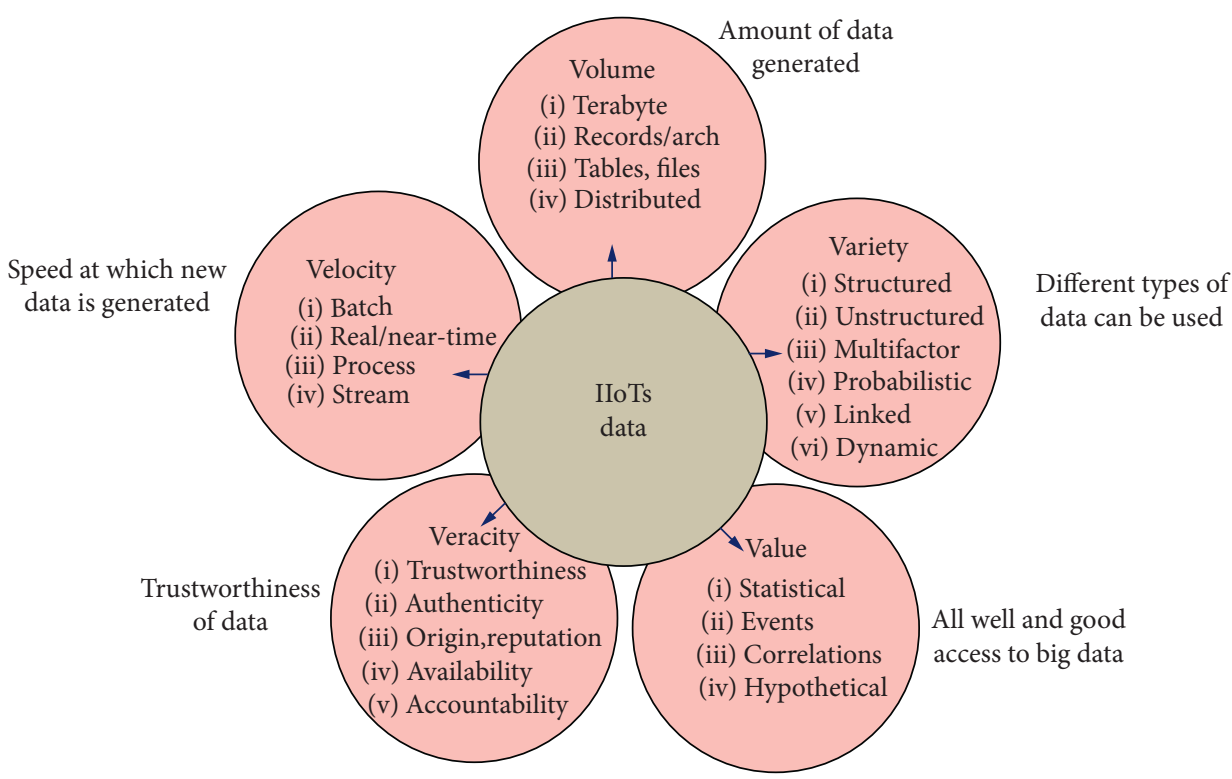

Figure 1: $5 \mathrm{Vs}$ of big data.

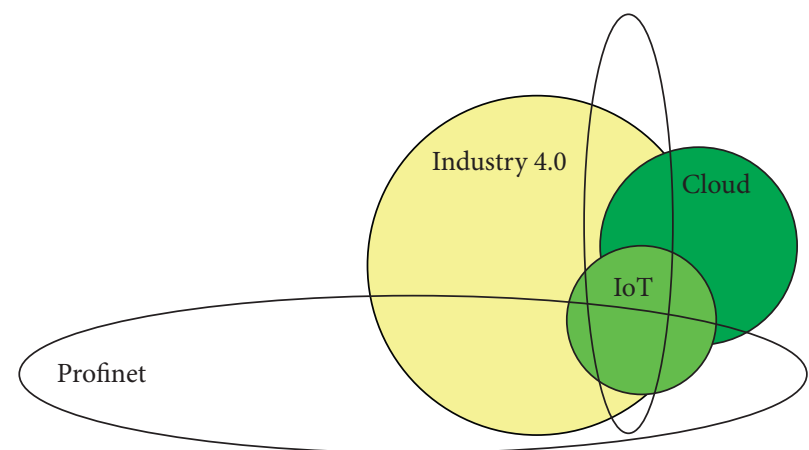

FIGURE 2: Generic IIoT.

which firstly studies the IoT developments and technologies related to cloud computing and smart cities and then focussed on the IoT technologies and cloud computing. Urquhart and Mcauley [19] presented an approach for the risks which lie for IioT, drawn both on the regulatory and technical perspectives. Gierej [20] presented the idea of business model for the companies implementing IIoT technologies. The approach is developed to help traditional companies in the transition of the digital market. Dachyar et al. [21] conducted in-depth analysis of the 26420 papers published in the area of IoT.

\section{Research Method}

Various taxonomies such as analytics tools, data sources, analytics techniques, industrial analytics applications, and requirements are used in the literature for analysing the data. Figure 1 shows the $5 \mathrm{Vs}$ of big data.

Figure 2 shows the generic IIoT.

The proposed method has used the Analytic Network Process for the evaluation of role of big data in IIoT. The ANP has several applications in different areas [22-25]. The
ANP approach was selected for evaluating the role of IIoT due to the reasons as this method works very well in situation, where complexity of dependencies exists among criteria and alternatives. The ANP method consists of three parts: (a) the goal, (b) criteria, and (c) alternatives. Five criteria along with subcriteria and three conditions were available as alternatives. The details regarding the ANP can be found in saaty (1996), while the following are the main steps of the ANP:

(a) Division of problem into subcriteria

(b) Quantitative scale of measure between 1 and 9

(c) Pairwise comparison for criteria and alternatives to achieve goal

(d) Eigenvalue and the related Eigenvector of the comparison matrix

(e) The consistency of matrix is measured

The "Consistency Index (CI)" and "Consistency Random (CR)" of pairwise comparison matrix are computed by equations (1) and (2): 
TABLE 1: Random consistency index.

\begin{tabular}{ccccccccccc}
\hline & 1 & 2 & 3 & 4 & 5 & 6 & 7 & 8 & 9 & 10 \\
\hline RI & 0 & 0 & 0.58 & 0.9 & 1.12 & 1.24 & 1.32 & 1.41 & 1.45 & 1.49 \\
\hline
\end{tabular}

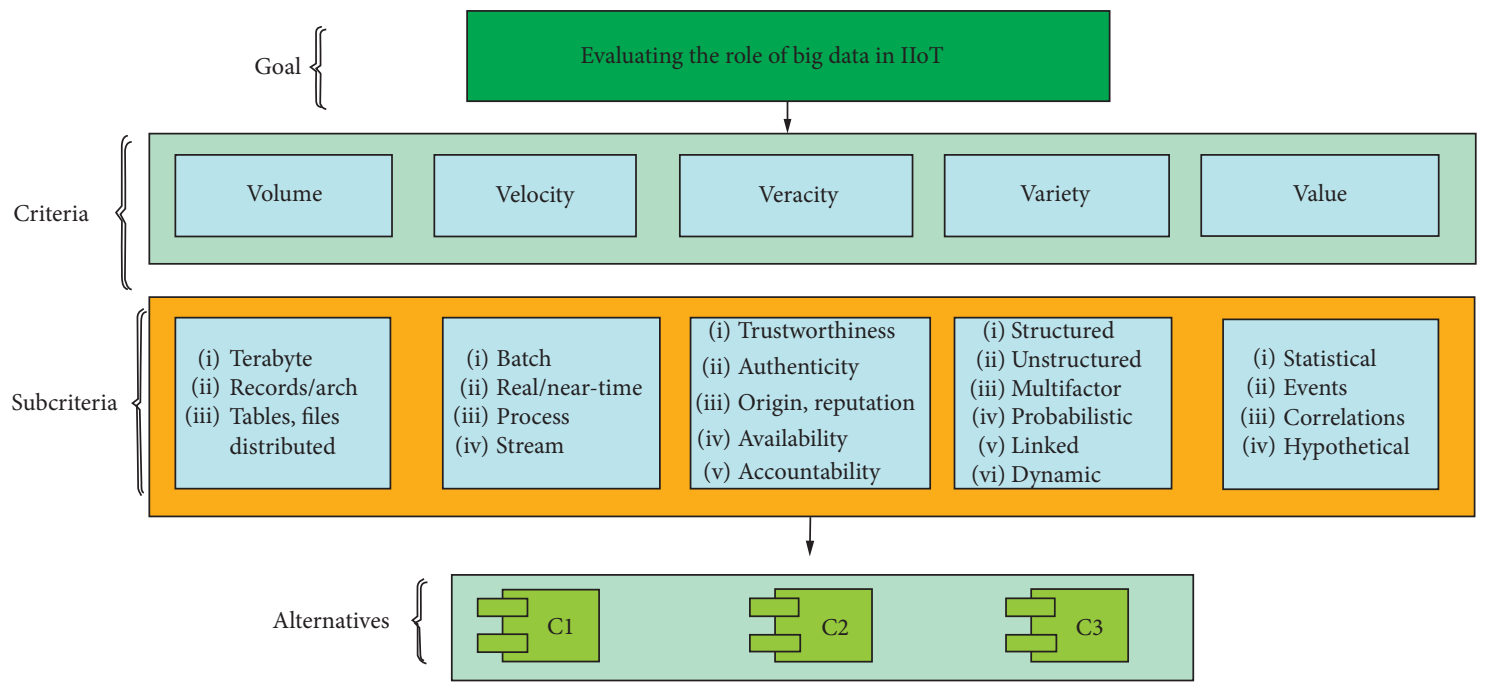

FIgURE 3: Goal, criteria, and alternatives.

TABle 2: Comparison with respect to condition 1.

\begin{tabular}{lcccccc}
\hline & Volume & Variety & Value & Veracity & Velocity & E.V \\
\hline Volume & 1 & 3 & 2 & 5 & 4 & 0.417 \\
Variety & $1 / 3$ & 1 & 2 & 3 & 2 & 0.219 \\
Value & $1 / 2$ & $1 / 2$ & 1 & 3 & 3 & 0.196 \\
Veracity & $1 / 5$ & $1 / 3$ & $1 / 3$ & 1 & 2 & 0.091 \\
Velocity & $1 / 4$ & $1 / 2$ & $1 / 3$ & $1 / 2$ & 1 & 0.077 \\
\hline
\end{tabular}

$\mathrm{CR}=0.065$.

TABLE 3: Comparison with respect to condition 2.

\begin{tabular}{lcccccc}
\hline & Volume & Variety & Value & Veracity & Velocity & E.V \\
\hline Volume & 1 & 2 & 3 & 2 & 5 & 0.383 \\
Variety & $1 / 2$ & 1 & 2 & 3 & 2 & 0.246 \\
Value & $1 / 3$ & $1 / 2$ & 1 & 2 & 3 & 0.172 \\
Veracity & $1 / 2$ & $1 / 3$ & $1 / 2$ & 1 & 2 & 0.124 \\
Velocity & $1 / 5$ & $1 / 2$ & $1 / 3$ & $1 / 2$ & 1 & 0.076 \\
\hline
\end{tabular}

$\mathrm{CR}=0.054$.

$$
\begin{gathered}
C_{i}=\frac{\lambda_{\max }-n}{n-1}, \\
\mathrm{CR}=\frac{\mathrm{CI}}{\mathrm{RI}} .
\end{gathered}
$$

Random consistency (RI) table is given by satty and is shown in Table 1 [26].

(f) Construction of weighted matrix

(g) Conversion of weighted super matrix into limit matrix
TABle 4: Comparison with respect to condition 3.

\begin{tabular}{lcccccc}
\hline & Volume & Variety & Value & Veracity & Velocity & E.V \\
\hline Volume & 1 & 5 & 2 & 3 & 7 & 0.453 \\
Variety & $1 / 5$ & 1 & 2 & 2 & 3 & 0.196 \\
Value & $1 / 2$ & $1 / 2$ & 1 & 3 & 2 & 0.184 \\
Veracity & $1 / 3$ & $1 / 2$ & $1 / 3$ & 1 & 2 & 0.103 \\
Velocity & $1 / 7$ & $1 / 3$ & $1 / 2$ & $1 / 2$ & 1 & 0.063 \\
\hline
\end{tabular}

$\mathrm{CR}=0.095$.

(h) Decision of the alternatives

Figure 3 shows the goal, criteria, and alternatives of the proposed research.

\section{Results and Discussion}

The process of pairwise comparisons of the proposed study was carried out in order to decide about available alternatives. The experts from the industry involved to check the contents against the available alternatives and relevant score should be given. Table 2 shows the pairwise comparison with respect to condition 1 for the available criteria.

Table 3 shows the pairwise comparison with respect to condition 2 for the available criteria.

Table 4 shows the pairwise comparison with respect to condition 3 for the available criteria.

Table 5 shows the pairwise comparison with respect to criteria (volume) for the available alternatives (conditions).

Table 6 shows the pairwise comparison with respect to criteria (variety) for the available alternatives (conditions).

Table 7 shows the pairwise comparison with respect to criteria (value) for the available alternatives (conditions). 
TABle 5: Comparison with respect to volume.

\begin{tabular}{lccrr}
\hline & Condition 1 & Condition 2 & Condition 3 & E.V \\
\hline Condition 1 & 1 & 3 & 4 & 0.623 \\
Condition 2 & $1 / 3$ & 1 & 2 & 0.239 \\
Condition 3 & $1 / 4$ & $1 / 2$ & 1 & 0.137 \\
\hline
\end{tabular}

$\mathrm{CR}=0.22$.

TABle 6: Comparison with respect to variety.

\begin{tabular}{lcccr}
\hline & Condition 1 & Condition 2 & Condition 3 & E.V \\
\hline Condition 1 & 1 & 2 & 2 & 0.490 \\
Condition 2 & $1 / 2$ & 1 & 2 & 0.312 \\
Condition 3 & $1 / 2$ & $1 / 2$ & 1 & 0.198 \\
\hline
\end{tabular}

$\mathrm{CR}=0.052$

TABLE 7: Comparison with respect to value.

\begin{tabular}{lcccr}
\hline & Condition 1 & Condition 2 & Condition 3 & E.V \\
\hline Condition 1 & 1 & 4 & 2 & 0.579 \\
Condition 2 & $1 / 4$ & 1 & 1 & 0.187 \\
Condition 3 & $1 / 2$ & 1 & 1 & 0.234 \\
\hline
\end{tabular}

$\mathrm{CR}=0.069$.

TABLE 8: Comparison with respect to veracity.

\begin{tabular}{lcccr}
\hline & Condition 1 & Condition 2 & Condition 3 & E.V \\
\hline Condition 1 & 1 & 3 & 4 & 0.623 \\
Condition 2 & $1 / 3$ & 1 & 2 & 0.239 \\
Condition 3 & $1 / 4$ & $1 / 2$ & 1 & 0.137 \\
\hline
\end{tabular}

$\mathrm{CR}=0.022$.

TABle 9: Comparison with respect to velocity.

\begin{tabular}{lcccc}
\hline & Condition 1 & Condition 2 & Condition 3 & E.V \\
\hline Condition 1 & 1 & 2 & 3 & 0.525 \\
Condition 2 & $1 / 2$ & 1 & 3 & 0.334 \\
Condition 3 & $1 / 3$ & $1 / 3$ & 1 & 0.142 \\
\hline
\end{tabular}

$\mathrm{CR}=0.056$.

TABLE 10: Weighted super matrix.

\begin{tabular}{lccccccccc}
\hline \multirow{2}{*}{ Node lable } & & \multicolumn{3}{c}{ IIoTs big data } & \multicolumn{4}{c}{ Alternatives } \\
& & Volume & Variety & Value & Veracity & Velocity & Condition 1 & Condition 2 & Condition 3 \\
\hline \multirow{3}{*}{ IIoTs big data } & Volume & 0.000 & 0.000 & 0.000 & 0.000 & 0.000 & 0.417 & 0.383 \\
& Variety & 0.000 & 0.000 & 0.000 & 0.000 & 0.000 & 0.219 & 0.246 & 0.453 \\
& Value & 0.000 & 0.000 & 0.000 & 0.000 & 0.000 & 0.196 & 0.172 & 0.196 \\
& Veracity & 0.000 & 0.000 & 0.000 & 0.000 & 0.000 & 0.091 & 0.124 & 0.103 \\
& Velocity & 0.000 & 0.000 & 0.000 & 0.000 & 0.000 & 0.077 & 0.076 & 0.063 \\
\hline \multirow{3}{*}{ Alternatives } & Condition 1 & 0.623 & 0.490 & 0.579 & 0.623 & 0.525 & 0.000 & 0.000 & 0.000 \\
& Condition 2 & 0.239 & 0.312 & 0.187 & 0.239 & 0.334 & 0.000 & 0.000 & 0.000 \\
& Condition 3 & 0.137 & 0.198 & 0.234 & 0.137 & 0.142 & 0.000 & 0.000 & 0.000 \\
\hline
\end{tabular}


TABLE 11: Limit matrix.

\begin{tabular}{lccccccccc}
\hline \multirow{2}{*}{ Node lable } & & \multicolumn{3}{c}{ IIoTs big data } & \multicolumn{4}{c}{ Alternatives } \\
& & Volume & Variety & Value & Veracity & Velocity & Condition 1 & Condition 2 & Condition 3 \\
\hline \multirow{3}{*}{ IIoTs big data } & Volume & 0.41 & 0.41 & 0.41 & 0.41 & 0.41 & 0.00 & 0.00 & 0.00 \\
& Variety & 0.22 & 0.22 & 0.22 & 0.22 & 0.22 & 0.00 & 0.00 & 0.00 \\
& Value & 0.19 & 0.19 & 0.19 & 0.19 & 0.19 & 0.00 & 0.00 & 0.00 \\
& Veracity & 0.10 & 0.10 & 0.10 & 0.10 & 0.10 & 0.00 & 0.00 & 0.00 \\
& Velocity & 0.07 & 0.07 & 0.07 & 0.07 & 0.07 & 0.00 & 0.00 & 0.00 \\
\hline \multirow{3}{*}{ Alternatives } & Condition 1 & 0.00 & 0.00 & 0.00 & 0.00 & 0.00 & 0.58 & 0.58 & 0.58 \\
& Condition 2 & 0.00 & 0.00 & 0.00 & 0.00 & 0.00 & 0.25 & 0.25 & 0.25 \\
& Condition 3 & 0.00 & 0.00 & 0.00 & 0.00 & 0.00 & 0.17 & 0.17 & 0.17 \\
\hline
\end{tabular}

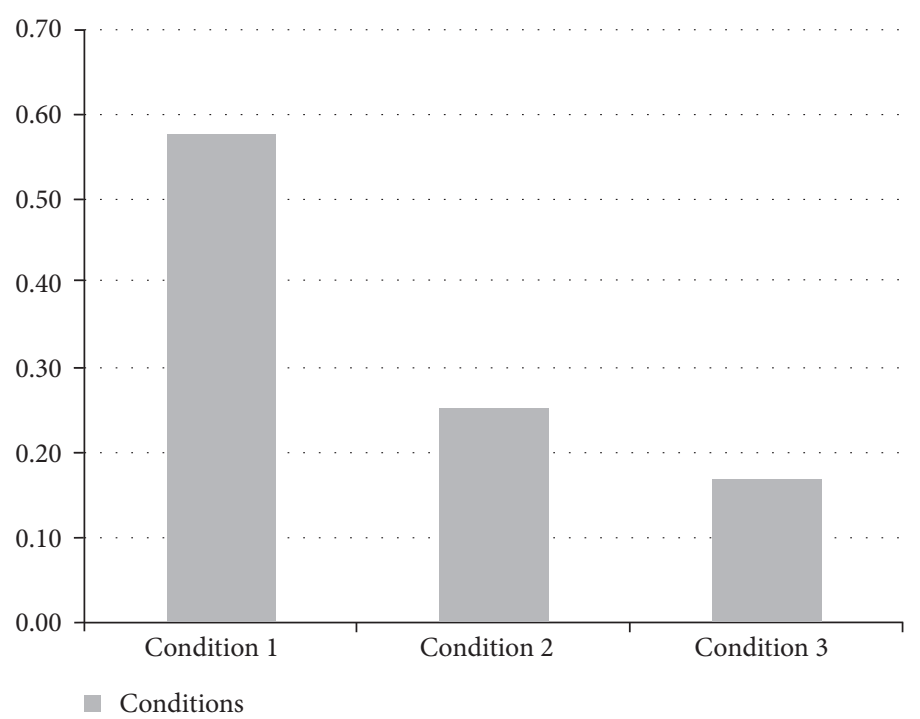

FIgURE 4: Ranking of available alternatives.

Table 8 shows the pairwise comparison with respect to criteria (veracity) for the available alternatives (conditions).

Table 9 shows the pairwise comparison with respect to criteria (velocity) for the available alternatives (conditions).

After pairwise comparisons, the E.V values of all the tables were merged into the weighted matrix. Table 10 shows the weighted matrix.

The weighted matrix (Table 10) was converted into the limit matrix in order to get the most appropriate condition in the available alternatives. Table 11 shows the limit matrix of the proposed study.

From limit matrix (Table 11), it is concluded that condition 1 is the best option among the available alternatives followed by condition 2, and then condition 3 . Figure 4 shows the decision of the available alternatives with their rank.

\section{Conclusion}

In recent years, the accessing and processing of big data become a challenging issue due to the limited storage space, computational time, networking, and IoT device end. IoT and big data are well thought-out to be the key concepts when describing new information architecture projects. With the enhancements and development in the Internet of Things and sensor deployments, the production of big data in Industrial Internet of Things is increased. Researchers are using different approaches and techniques for evaluating the role of big data in IIoT. These techniques are not handling the situations when complexity of dependency arises among parameters of the alternatives. The contribution of the proposed research is to use the approach of Analytic Network Process for evaluating the role of big data in IIoT. The process of pairwise comparison was carried out for the criteria and available alternatives. The results show that the proposed research works well for evaluating the role of big data in IIoT.

\section{Data Availability}

No data are available

\section{Conflicts of Interest}

The authors declare that they have no conflicts of interest. 


\section{Acknowledgments}

This work was supported by Science and Technology Project of State Grid Xizang Electric Power Co., Ltd. (SGXZJY00JHJS2000007), Influence of Energy Storage Technology Application on Power Grid, and Science and Technology Project of State Grid Zizang Electric Power Co., Ltd. (SGXZJY00JHJS2000008), Research Technology Service of Multi Energy Complementary Demonstration Application.

\section{References}

[1] B. Diã"Ne, J. J. P. C. Rodrigues, O. Diallo, E. L. H. M. Ndoye, and V. V. Korotaev, "Data management techniques for Internet of Things," Mechanical Systems and Signal Processing, vol. 138, Article ID 106564, 2020.

[2] M. Younan, E. H. Houssein, M. Elhoseny, and A. A. Ali, "Challenges and recommended technologies for the industrial Internet of Things: a comprehensive review," Measurement, vol. 151, Article ID 107198, 2020.

[3] M. H. Ur Rehman, I. Yaqoob, K. Salah, M. Imran, P. P. Jayaraman, and C. Perera, "The role of big data analytics in industrial Internet of Things," Future Generation Computer Systems, vol. 99, pp. 247-259, 2019.

[4] M. S. Hadj Sassi, F. G. Jedidi, and L. C. Fourati, "A new architecture for cognitive Internet of Things and big data," Procedia Computer Science, vol. 159, pp. 534-543, 2019.

[5] P. Sunhare, R. R. Chowdhary, and M. K. Chattopadhyay, "Internet of Things and data mining: an application oriented survey," Journal of King Saud University-Computer and Information Sciences, 2020.

[6] C. Yao, S. Wu, Z. Liu, and P. Li, “A deep learning model for predicting chemical composition of gallstones with big data in medical Internet of Things," Future Generation Computer Systems, vol. 94, pp. 140-147, 2019.

[7] W. Z. Khan, M. H. Rehman, H. M. Zangoti, M. K. Afzal, N. Armi, and K. Salah, "Industrial Internet of Things: recent advances, enabling technologies and open challenges," Computers \& Electrical Engineering, vol. 81, Article ID 106522, 2020.

[8] N. Gulati and P. D. Kaur, "Towards socially enabled internet of industrial things: architecture, semantic model and relationship management," Ad Hoc Networks, vol. 91, Article ID 101869, 2019.

[9] G. Aceto, V. Persico, and A. Pescapé, "Industry 4.0 and health: Internet of Things, big data, and cloud computing for healthcare 4.0," Journal of Industrial Information Integration, vol. 18, Article ID 100129, 2020.

[10] Y. Karaca, M. Moonis, Y.-D. Zhang, and C. Gezgez, "Mobile cloud computing based stroke healthcare system," International Journal of Information Management, vol. 45, pp. 250261, 2019.

[11] S. H. Almotiri, M. A. Khan, and M. A. Alghamdi, "Mobile health ( $m$-health) system in the context of IoT," in Proceedings of the 2016 IEEE 4th International Conference on Future Internet of Things and Cloud Workshops (FiCloudW), pp. 39-42, Vienna, Austria, August 2016.

[12] B. Feldner and P. Herber, "A qualitative evaluation of IPv6 for the industrial Internet of Things," Procedia Computer Science, vol. 134, pp. 377-384, 2018.
[13] H. Boyes, B. Hallaq, J. Cunningham, and T. Watson, "The industrial internet of things (IIoT): an analysis framework," Computers in Industry, vol. 101, pp. 1-12, 2018.

[14] K. Alexopoulos, S. Koukas, N. Boli, and D. Mourtzis, "Architecture and development of an industrial internet of things framework for realizing services in industrial product service systems," Procedia CIRP, vol. 72, pp. 880-885, 2018.

[15] M. Ge, H. Bangui, and B. Buhnova, "Big data for Internet of Things: a survey," Future Generation Computer Systems, vol. 87, pp. 601-614, 2018.

[16] Y. J. Lin, C.-B. Lan, and C.-Y. Huang, "A realization of cyberphysical manufacturing control system through Industrial Internet of Things," Procedia Manufacturing, vol. 39, pp. 287-293, 2019.

[17] M. Humayun, N. Z. Jhanjhi, A. Alsayat, and V. Ponnusamy, "Internet of Things and ransomware: evolution, mitigation and prevention," Egyptian Informatics Journal, 2020.

[18] D. Jiang, "The construction of smart city information system based on the Internet of Things and cloud computing," Computer Communications, vol. 150, pp. 158-166, 2020.

[19] L. Urquhart and D. Mcauley, "Avoiding the internet of insecure industrial things," Computer Law \& Security Review, vol. 34, no. 3, pp. 450-466, 2018.

[20] S. Gierej, "The framework of business model in the context of Industrial Internet of Things," Procedia Engineering, vol. 182, pp. 206-212, 2017.

[21] M. Dachyar, T. Y. M. Zagloel, and L. R. Saragih, "Knowledge growth and development: internet of things (IoT) research, 2006-2018," Heliyon, vol. 5, no. 8, Article ID e02264, 2019.

[22] S. Nazir, S. Shahzad, I. Zada, and H. Khan, "Evaluation of software birthmarks using fuzzy analytic hierarchy process," in Proceedings of the Fourth International Multi-Topic Conference, pp. 171-175, Jamshoro, Pakistan, Decembe 2015.

[23] S. Nazir, S. Shahzad, Z. Hussain, M. Iqbal, and A. Keerio, "Evaluating student grades using analytic network process," Sindh University Research Journal (Science Series), vol. 47, pp. 1-5, 2015.

[24] S. Nazir, S. Anwar, S. A. Khan et al., "Software component selection based on quality criteria using the analytic network process," Abstract and Applied Analysis, vol. 2014, Article ID 535970, 12 pages, 2014.

[25] S. Nazir, S. Shahzad, M. Nazir, and H. U. Rehman, "Evaluating security of software components using analytic network process," in Proceedings of the 11th International Conference on Frontiers of Information Technology (FIT), IEEE, Islamabad, Pakistan, pp. 183-188, 2013.

[26] T. L. Saaty, "Relative measurement and its generalization in decision making why pairwise comparisons are central in mathematics for the measurement of intangible factors the analytic hierarchy/network process," Revista de la Real Academia de Ciencias Exactas, Fisicas y Naturales. Serie A. Matematicas, vol. 102, no. 2, pp. 251-318, 2008. 